\title{
Dünnschichtchromatographische Trennung oraler Kontrazeptiva neben einigen Sexualsteroiden
}

\author{
Von I. Székács und M. KLembala \\ Aus der Biocbemiscben Abteilung des Staatl. Instituts für Hygiene, Budapest \\ (Eingegangen am 1. Juli 1969)
}

Eine zweidimensionale dünnschichtchromatographische Methode wird beschrieben, dic das Trennen und Identifizieren einer Anzahi oraler Kontrazeptiva und verwandter Steroide ermöglicht.

\section{Thin layer chromatographic separation of oral contraceptives and some sex steroids}

A two dimensional thin layer chromatographic method is described for the separation and identification of a number of oral contraceptives and related steroids.

Zur Analyse oraler Kontrazeptiva sind in der Fachliteratur nur wenige Verfahren bekannt. ZANDER und Mitarbeiter (1) sowie GraEF und STAUDINGER (2) berichten über die Trennung und Bestimmung verschiedener Steroide. Eine dünnschichtchromatographische $\mathrm{Me}$ thode zur Analyse oraler Kontrazeptiva und ihrer therapeutisch angewandten Komponenten wurde unlängst von RöDER veröffentlicht (3).

Im Laufe unserer Untersuchungen erwies es sich als notwendig, einige Steroide, darunter auch orale Kontrazeptiva, in biologischem Material zu trennen bzw. zu identifizieren. Um dies $z u$ erreichen, wurde zuerst das RöDER sche Verfahren herangezogen. Da der erwünschte .Trenneffekt nicht erreicht wurde, waren wir gezwungen, die Methode zu modifizieren, wobei eine ausgezeichnete Trennung der zu untersuchenden Stoffe erzielt werden konnte. Die Modifikation besteht im wesentlichen darin, daß wir die zwei von RöDER angegebenen Fließmittelsysteme mit einem dritten, bestehend aus Chloroform und Aceton, ergänzten. Die drei verschiedenen Systeme werden entweder simultan oder zu zweit, d.h. ein RöDERsches, kombiniert mit dem von uns vorgeschlagenen System, zweidimensional zur Anwendung gebracht.

\section{Methodik}

Die folgenden Substanzen ${ }^{1}$ ) wurden untersucht:

Progesteron, Mestranol, Norethinodrel und Ethynodioldiacetat (Arzneimittelfabrik Köbánya, Budapest, Ungarn)

Mestranol und Lynestrenol (N. V. „Organon“, Oss, Holland)

1) Progesteron $=\Delta 4-$ Pregnen-3,20-dion, Mestranol $=\Delta 1,3,5$ (10)-3-Methoxy-19-Nor-17 $\alpha$-pregnatrien-20-in-17-ol, Norethinodrel $=\Delta 5(10)$-19-Nor-17 $\alpha$-pregnen-20-in-3, 17-diol, Ethynodioldiacetat $=3 \beta, 17 \alpha$-Diacetoxy- $\Delta 419$-nor-pregnen-20-in, Lynestre$\mathrm{nol}=\Delta 4-19-$ Nor-17 $\alpha$-pregnen-20-in-17-ol, Cyproteron $=1,2 \alpha-\mathrm{Me}-$ thylen- 6 chloro- $\Delta 4,6$-pregnadien-17 $\alpha$-ol-3,20-dion, Chlormadinonacetat $=\Delta 4-6$-Chlor-6-dehydro-17 $\alpha$-acetoxy-pregnen-3,20-dion, Infecundin $=0,1 \mathrm{mg}$ Mestranol $+2,5 \mathrm{mg}$ Norethinodrel pro Tablette, Bisecurin $=1 \mathrm{mg}$ Ethynodioldiacetat $+0,1 \mathrm{mg}$ Mestranol pro Tablette, Lyndiol $=2,5 \mathrm{mg}$ Lynestrenol $+0,075 \mathrm{mg}$ Mestranol pro Tablette, C-Queens I = 0,08 mg Mestranol pro (weiße) Tablette, C-Queens $I I=0,08 \mathrm{mg}$ Mestranol $+2 \mathrm{mg}$ Chlormadinon-acetat pro (gelbe) Tablette, Enavid = 2,5 mg Norethinodrel + 0,1 $\mathrm{mg}$ Mestranol pro Tablette, Orgametril $=5 \mathrm{mg}$ Lynestrenol pro Tablette.
Cyproteron und Cyproteronacetat (Schering AG, Berlin) Östron, Östradiol, Östradioldipropionat (Arzneimittelfabrik Köbánya, Budapest, Ungarn)

Die oralen Kontrazeptiva:

Infecundin und Bisecurin (Arzneimittelfabrik Köbánya, Budapest, Ungarn)

Lyndiol (N. V. „,Organon“, Oss, Holland)

C-Queens I.- und II. (Lilly and Comp., USA)

Enavid (Searle, England).

Alle Substanzen - mit Ausnahme von Óstradiol - wurden in frisch destilliertem Chloroform p. a., das Ostradiol in einem Gemisch von Chloroform-Dioxan 1:1 gelöst. In einigen Fällen benutzten wir als Lösungsmittel anstatt Chloroform Dichlormethan; die Verschiedenheit der Lösungsmittel verursachte weder in den $R_{F}$-Werten noch in den nach der Sichtbarmachung entstandenen Farben einen Unterschied.

Zur Analyse der Kontrazeptiva wurden 2-5 Tabletten mit der gleichen Menge Seesand fein verrieben und viermal mit 2,5 m/frisch destilliertem Chloroform p. a. extrahiert. Die in einem Destillierkolben vereinigten Extrakte wurden im Vakuum bei Raumtemperatur unter $\mathrm{N}_{2}$-Gasstrom eingedampt und die von Lösungsmittelresten vollkommen befreiten Rückstände in Chloroform oder Dichlormethan aufgenommen. Aus dieser Lösung wurden sodann die benötigten Mengen auf Dünnschichtplatten aufgetragen.

$20 \times 20 \mathrm{~cm}$ Glasplatten wurden mit einer Kieselgel-G-WasserSuspension ( $24 \mathrm{~g}$ Kieselgel in $48 \mathrm{~m} /$ Wasser für 4 Platten) mit Hilfe eines "Desaga“-Streichgeräts bestrichen, zur Aktivierung bei $110^{\circ}$ im Trockenschrank $30 \mathrm{Min}$. erhitzt und nachfolgend 24 Stdn. bei Raumtemperatur aufbewahrt. Unmittelbar vor der Anwendung wurden die Platten für $10 \mathrm{Min}$. in einen Trockenschrank gestellt und nach Abkühlen mit den zu untersuchenden Substanzen beschickt.

Die aufsteigende Entwicklung wurde in Trennkammern durchgeführt, die mit Filterpapier ausgekleidet und 1 Std. lang mit dem Fließmittel gesättigt waren.

Folgende Fließmittel wurden angewendet:

a) Athylacetat-Cyclohexan-Aceton 25:75:10 (v/v)

b) Äthylacetat-Cyclohexan $54: 46(v / v)$

c) Chloroform-Aceton 90:10 (v/v).

Zur zweidimensionalen Chromatographic erwiesen sich die folgenden Kombinationen als geeignete Systeme:

I. Fließmittel a) - Fließmittel b)

II. Fließmittel c) - Fließmittel a)

III. Fließmittel c) - Fließmittel b)

Die für die Chromatographie erforderliche Temperatur war $20-23^{\circ}$, die Laufzeit bis zu eincr Höhe von $15 \mathrm{~cm}$ betrug durchschnittlich $30-40 \mathrm{Min}$. 
Tab. 1

RF-Werte, Farben und Fluoreszenz der untersuchten Substanzen

\begin{tabular}{|c|c|c|c|c|c|c|c|c|c|c|c|c|c|c|c|}
\hline \multirow[t]{2}{*}{ Name } & \multirow[b]{2}{*}{$R_{\mathrm{F}}$} & \multicolumn{4}{|c|}{ 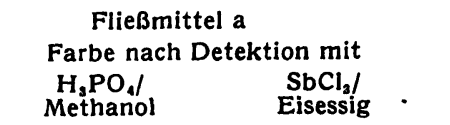 } & \multirow[b]{2}{*}{$R_{\mathbf{F}}$} & \multicolumn{4}{|c|}{$\begin{array}{l}\text { Fließmittel b } \\
\text { Farbe nach Detektion mit } \\
\mathrm{H}_{2} \mathrm{PO}_{4} / \\
\text { Methanol } \quad \mathrm{SbCl}_{2} / \\
\text { Eisessig }\end{array}$} & \multirow[b]{2}{*}{$R_{\mathrm{F}}$} & \multicolumn{4}{|c|}{ 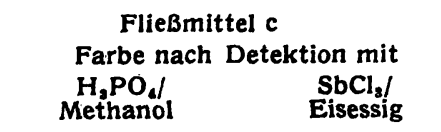 } \\
\hline & & $\begin{array}{l}\text { Tages- } \\
\text { licht }\end{array}$ & $\underset{\text { Licht }}{\text { UV- }}$ & $\begin{array}{l}\text { Tages- } \\
\text { licht }\end{array}$ & $\begin{array}{l}\text { UV- } \\
\text { Licht }\end{array}$ & & $\begin{array}{l}\text { Tages- } \\
\text { licht }\end{array}$ & UV- & $\begin{array}{l}\text { Tages- } \\
\text { licht }\end{array}$ & $\underset{\text { Licht }}{\text { UV- }}$ & & $\begin{array}{l}\text { Tages- } \\
\text { licht }\end{array}$ & $\begin{array}{l}\text { UV- } \\
\text { Licht }\end{array}$ & $\begin{array}{c}\text { Tages- } \\
\text { licht }\end{array}$ & UV. \\
\hline $\begin{array}{l}\text { Norethyno- } \\
\text { drel }\end{array}$ & 0,35 & $\begin{array}{l}\text { rötlich- } \\
\text { braun }\end{array}$ & braun & $\begin{array}{l}\text { rötlich- } \\
\text { braun }\end{array}$ & braun & 0,60 & $\begin{array}{l}\text { rötlich- } \\
\text { braun }\end{array}$ & braun & $\begin{array}{l}\text { rötlich- } \\
\text { braun }\end{array}$ & bordo & 0,48 & $\begin{array}{l}\text { rötlich-. } \\
\text { braun, }\end{array}$ & $\begin{array}{l}\text { rötlich- } \\
\text { braun }\end{array}$ & $\begin{array}{l}\text { rötlich- } \\
\text { braun }\end{array}$ & braun \\
\hline Mestranol & 0,45 & rot & $\begin{array}{l}\text { orange- } \\
\text { gelb }\end{array}$ & $\begin{array}{l}\text { violett- } \\
\text { rot }\end{array}$ & rot & 0,65 & rot & $\begin{array}{l}\text { orange- } \\
\text { gelb }\end{array}$ & $\begin{array}{l}\text { violett- } \\
\text { rot }\end{array}$ & rot & 0,54 & rot & $\begin{array}{l}\text { orange- } \\
\text { gelb }\end{array}$ & $\begin{array}{l}\text { violett- } \\
\text { rot }\end{array}$ & rot \\
\hline Lynestrenol & 0,53 & $\begin{array}{l}\text { gelblich- } \\
\text { braun }\end{array}$ & $\begin{array}{l}\text { gelblich- } \\
\text { braun }\end{array}$ & $\begin{array}{l}\text { bräun- } \\
\text { lich rot }\end{array}$ & $\begin{array}{l}\text { gelblich- } \\
\text { braun }\end{array}$ & 0,70 & $\begin{array}{l}\text { gelblich- } \\
\text { braun }\end{array}$ & - braun & $\begin{array}{l}\text { bräun- } \\
\text { lich rot }\end{array}$ & $\begin{array}{l}\text { rötlich- } \\
\text { braun }\end{array}$ & 0,58 & $\begin{array}{l}\text { gelblich- } \\
\text { bràun }\end{array}$ & $\begin{array}{l}\text { gelblich- } \\
\text { braun }\end{array}$ & $\begin{array}{l}\text { bräun- } \\
\text { lich rot }\end{array}$ & braun \\
\hline $\begin{array}{l}\text { Ethinodiol- } \\
\text { diacetat }\end{array}$ & 0,52 & $\begin{array}{l}\text { gelblich- } \\
\text { braun }\end{array}$ & $\begin{array}{l}\text { gelblich- } \\
\text { braun }\end{array}$ & $\begin{array}{l}\text { bräun- } \\
\text { lich rot }\end{array}$ & braun & 0,66 & $\begin{array}{l}\text { rötlich- } \\
\text { braun }\end{array}$ & $\begin{array}{l}\text { gelblich- } \\
\text { braun }\end{array}$ & - gelblich- & $\begin{array}{l}\text { gelblich- } \\
\text { braun }\end{array}$ & $0,7.3$ & $\begin{array}{l}\text { gelblich- } \\
\text { brạun }\end{array}$ & $\begin{array}{l}\text { gelblich- } \\
\text { braun }\end{array}$ & $\begin{array}{l}\text { rötlich- } \\
\text { violett }\end{array}$ & $\begin{array}{l}\text { rötlich- } \\
\text { braun }\end{array}$ \\
\hline $\begin{array}{l}\text { Chlormadi- } \\
\text { nonacetat }\end{array}$ & 0,27 & $\begin{array}{l}\text { türkis- } \\
\text { grün }\end{array}$ & grün & $\begin{array}{l}\text { türkis- } \\
\text { grün }\end{array}$ & grün & 0,51 & grün & grün & $\begin{array}{l}\text { türkis- } \\
\text { blau }\end{array}$ & grün & 0,59 & $\begin{array}{l}\text { grün- } \\
\text { lich- } \\
\text { blau }\end{array}$ & $\begin{array}{l}\text { türkis- } \\
\text { grün }\end{array}$ & $\begin{array}{l}\text { grün- } \\
\text { lich- } \\
\text { blau }\end{array}$ & grün \\
\hline Progesteron & 0,36 & farblos & $\begin{array}{l}\text { schwach } \\
\text { bläulich- } \\
\text { grün }\end{array}$ & farblos & $\begin{array}{l}\text { schwach } \\
\text { grün }\end{array}$ & 0,53 & farblos & $\begin{array}{l}\text { schwach } \\
\text { blau }\end{array}$ & farblos & $\begin{array}{l}\text { schwach } \\
\text { grün }\end{array}$ & 0,56 & farblos & $\begin{array}{l}\text { schwach } \\
\text { blau }\end{array}$ & farblos & $\begin{array}{l}\text { grün- } \\
\text { lich- } \\
\text { blau }\end{array}$ \\
\hline Ostron & 0,31 & gelb & $\begin{array}{l}\text { schwach } \\
\text { grünlich- } \\
\text { gelb }\end{array}$ & rosa & $\begin{array}{l}\text { zitro- } \\
\text { nengelb }\end{array}$ & 0,57 & gelb & $\begin{array}{l}\text { schwach } \\
\text { grünlich- } \\
\text { gelb. }\end{array}$ & rosa & $\begin{array}{l}\text { orange- } \\
\text { gelb }\end{array}$ & 0,48 & $\begin{array}{l}\text { zitro- } \\
\text { nengelb }\end{array}$ & $\begin{array}{l}\text { schwach } \\
\text { grünlich- } \\
\text { gelb }\end{array}$ & & gelb \\
\hline Ostradiol & 0,23 & gelb & $\begin{array}{l}\text { schwach } \\
\text { grünlich- } \\
\text { gelb }\end{array}$ & rosa & $\begin{array}{l}\text { orange- } \\
\text { gelb }\end{array}$ & 0,49 & $\begin{array}{l}\text { orange- } \\
\text { gelb }\end{array}$ & $\begin{array}{l}\text { schwach } \\
\text { gelblich- } \\
\text { grün }\end{array}$ & rosa & $\begin{array}{l}\text { orange- } \\
\text { gelb }\end{array}$ & 0,26 & $\begin{array}{l}\text { zitro- } \\
\text { nengelb }\end{array}$ & $\begin{array}{l}\text { schwach } \\
\text { grünlich } \\
\text { gelb }\end{array}$ & $\begin{array}{l}\text { gelbulich- } \\
\text { rosa }\end{array}$ & gelb \\
\hline $\begin{array}{l}\text { Ostradioldi- } \\
\text { propionat }\end{array}$ & 0,63 & $\begin{array}{l}\text { zitro- } \\
\text { nengelb }\end{array}$ & $\begin{array}{l}\text { schwach } \\
\text { grün }\end{array}$ & rosa & $\begin{array}{l}\text { zitro- } \\
\text { nengelb }\end{array}$ & 0,75 & gelb & $\begin{array}{l}\text { schwach } \\
\text { gelblich= } \\
\text { grün }\end{array}$ & $\begin{array}{l}\text { gelblich- } \\
=\text { rot }\end{array}$ & gelb & 0,74 & $\begin{array}{l}\text { zitro- } \\
\text { nengelb }\end{array}$ & $\begin{array}{l}\text { schwach } \\
\text { grün }\end{array}$ & $\begin{array}{l}\text { gelblich- } \\
\text { rosa }\end{array}$ & $\begin{array}{l}\text { zitro- } \\
\text { nengelb }\end{array}$ \\
\hline Cyproteron & 0,22 & $\begin{array}{l}\text { rötlich- } \\
\text { braun }\end{array}$ & $\begin{array}{l}\text { bräun- } \\
\text { lichgrün }\end{array}$ & rot & braun & 0,45 & $\begin{array}{l}\text { rötlich- } \\
\text { braun }\end{array}$ & braun & rot & braun & 0,39 & braun & braun & blau & braun \\
\hline $\begin{array}{l}\text { Cyproteron- } \\
\text { acetat }\end{array}$ & 0,23 & $\begin{array}{l}\text { bläulich- } \\
\text { grün }\end{array}$ & $\begin{array}{l}\text { - türkis- } \\
\text { blau }\end{array}$ & $\begin{array}{l}\text { grünlich- } \\
\text { blau }\end{array}$ & - grün & 0,43 & blau & hellblau & $\begin{array}{l}\text { grünlich } \\
\text { blau }\end{array}$ & grün & 0,53 & blau & hellblau & $\begin{array}{l}\text { türkkis- } \\
\text { grünn }\end{array}$ & braun \\
\hline
\end{tabular}

Die bei Raumtemperatur getrockneten Platten wurden zur Sichtbarmachung mit einem der von RöDER verwendeten Sprühreagenzien

a) Phosphorsäure-Methanol $1: 1(v / v)$ oder

b) Antimon[III]-chlorid-Eisessig $1: 1(\mathrm{~g} / \mathrm{v})$ besprüht und 10 bis 20 Min. im Trockenschrank bei $110^{\circ}$ erhitzt. Die abgekühlten Platten wurden zunächst schwarz-weiß photographiert, sodann unter UV-Licht (Wood-Filter, Osram Hg-v 300, 75 W) gestellt. Die stark fluoreszierenden Flecken wurden ebenfalls schwarzweiß und nachfolgend auch auf farbige Emulsion (Orwo Color UT $16,5 \%$-CuSO ${ }_{4}$-Filter) photographiert. Die mit sorgfältig gereinigten Glasscheiben bedeckten Platten wurden an allen Seiten luftdicht mit Klebstreifen verklebt. Auf diese Weise konnten die farbigen Flecke längere Zeit konserviert werden.

\section{Ergebnisse}

Die Untersuchungsergebnisse sind in Tabelle 1 zusammengestellt.

Die in Tabelle 1 angegebenen Zahlen sind Durchschnittswerte von 24-156 Bestimmungen; die einzelnen Parallelbestimmungen weichen im $R_{\mathbf{F}}$-Wert voneinander höchstens um $\pm 0,03 R_{\mathrm{F}}$-Wert ab.

Aus Tabelle 1 ist zu ersehen, daß jede der von uns untersuchten Substanzen mit Hilfe von drei Charakteristika, nämlich $R_{\mathrm{F}}$-Wert, Farbe bei Tageslicht und Fluoreszenż, eindeutig charakterisiert werden kann. Selbst sich lich verhaltende Substanzen wie z. B. Lynestrenol und Ethynodioldiacetat, oder Cyproteron und Cyproteron- acetat, die im RöDERschen Fließmittelsystem gleichen $R_{\mathrm{F}}$-Wert, Farbe und Fluoreszenz aufweisen, können aufgrund unterschiedlicher $R_{\mathrm{F}}$-Werte in dem von uns modifizierten Fließmittelsystem einwandfrei getrennt werden.

Im UV-Licht sind $1 \mu \mathrm{g}$, meist aber noch geringeṛe Substanzmengen nachweisbar.

In Abbildung $1 \mathrm{a}-\mathrm{c}$ ist das Trennungsvermögen der drei zweidimensionalen Verfahren einander gegenüber gestellt.

Es zeigt sich, daß im Lösungsmittelsystem II. bzw. III. die einzelnen Steroide besser getrennt werden können als in dem von RöDER beschriebenen System I.

Die hohe Empfindlichkeit des Verfahrens ermöglicht die Anwendung zur Reinheitsprüfung von Handelspräparaten. Unsere Befunde zeigten z. B., daß das Produkt Enavid außer Norethynodrel und Mestranol noch andere gut nachweisbare, von uns jedoch nicht identifizierte Steroide enthält, während die Chromatogramme der Lyndiol und Orgametril-Tabletten nur kaum nachweisbare Spuren unbekannter Substanzen außer Mestranol und Lynestrenol bzw. Lynestrenol aufweisen.

Für das zur Verfügung gestellte Material sprechen wir den Firmen Atzneimittelfabrik Köbánya, Budapest; N. V. „Organon“", Oss; Searle, England, und Herrn Prof. F. NeumanN, Schering AG, Berlin, unseren aufrichtigen Dank aus. Für die technische Assistenz danken wir Herrn Valenjtin Varga. 


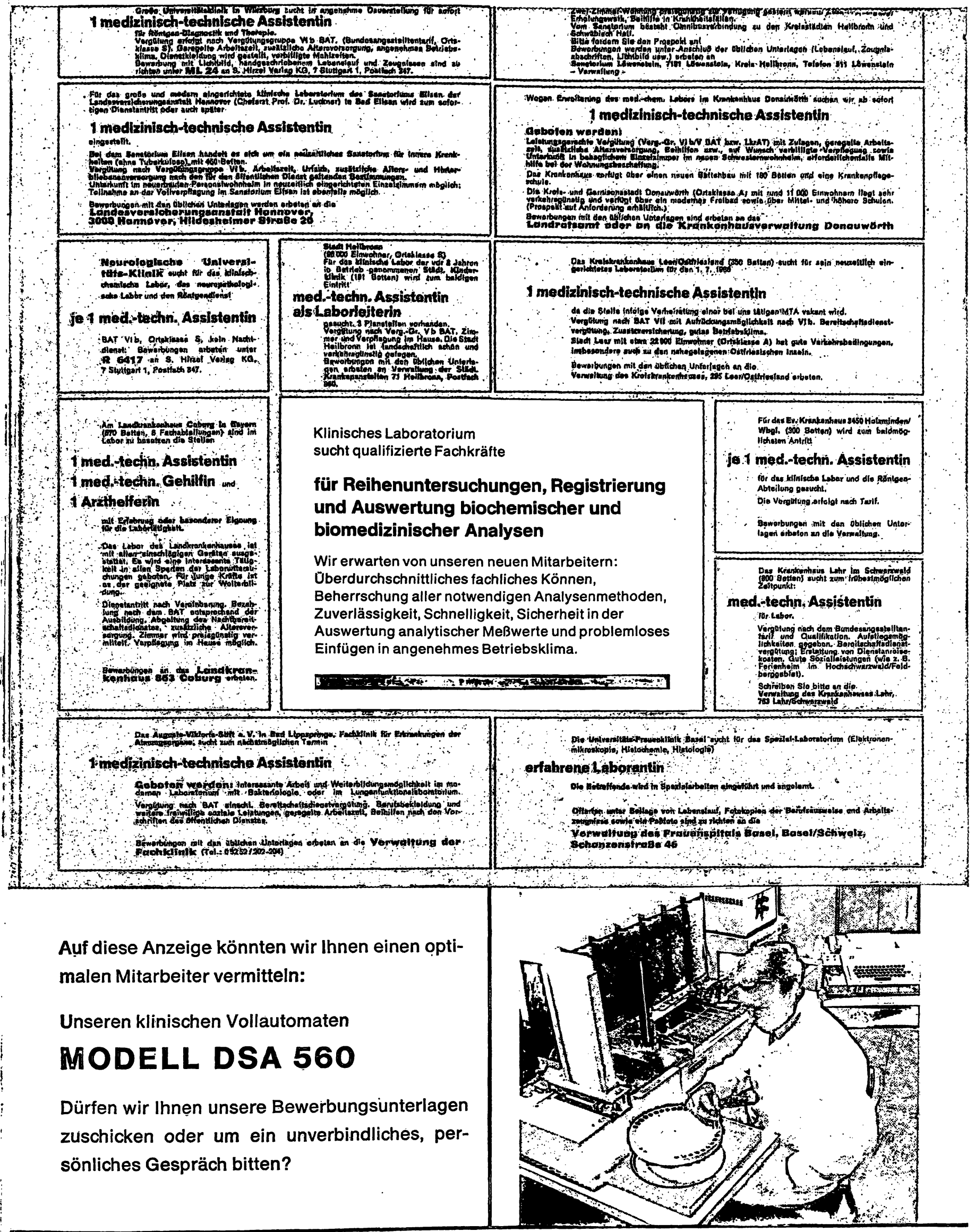

\section{Beckman}


Asmus

\section{Einführung}

in die Höhere Mathematik und ihre Anwendungen

Ein Hilfsbuch für Chemiker, Physiker und andere Naturwissenschaftler

Von Prof. Dr. Erik Asmus

5. Auflage. Oktav. Mit 184 Abbildungen. XII, 410 Seiten. 1969. Plastik flexibel DM 24,-

Wir wollen mit ganz besonderem Nachdruck darauf hinweisen, daß hier ein Buch vorliegt, das so seht in der Sprache des Naturwissenschaftlers geschrieben wird, $\mathrm{da} ß$ es für den Biologen, Chemiker oder Physiker in ganz besonderem Maße lesbar wird. Vom Stoff her vermittelt das Buch ausführlich und an Hand sehr vieler Beispiele aus der Praxis des Naturforschers die Differentialrechnung, und die Integralrechnung.

Die Natur, Stuttgart

Walter de Gruyter \& Co $\cdot$ Berlin

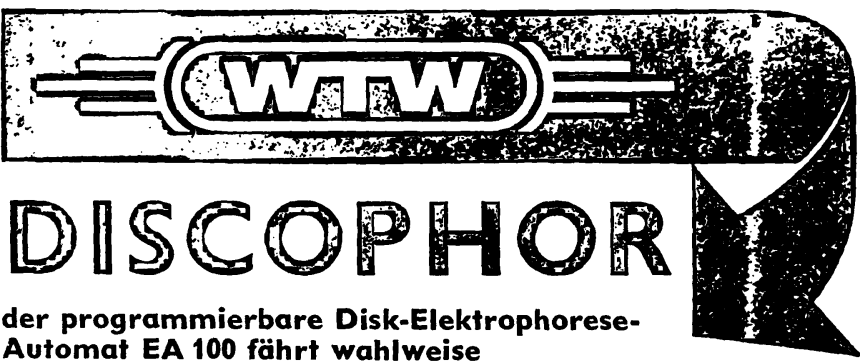

\section{- präparativ \\ - oder \\ - analytisch}

und liefert bei präparativer Verwendung automatisch

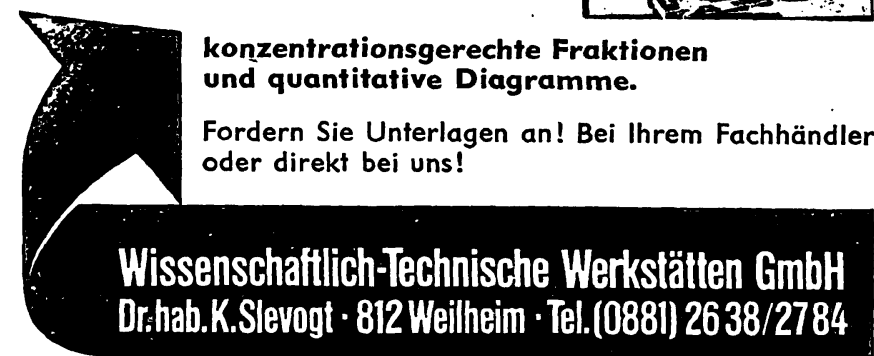

Verkaufbüros: Essen, Lönsberg 22, Tel. 510019

Dusslingen, W. Bohn, Farrenbergweg 5

Hagen, Hestertstraße 64, Tel. 45857

Bad Nauheim, Frankfurter Str. 39, Tel. 4860

\section{Aminosäure- Trennungen können Sie mit dieser Apparatur in 15 Minuten ausführen}

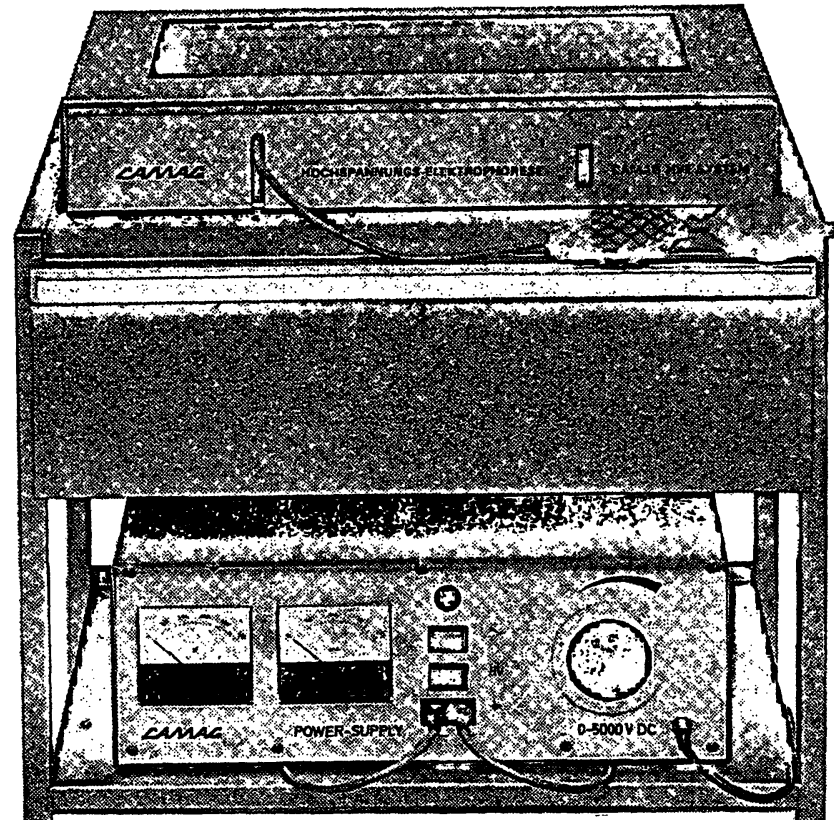

Es ist das neue CAMAG Hochspannungs-ElektrophoreseSystem mit seinem einzigartigen Kühlprinzip. Wollen Sie wissen, wie das funktioniert? Sie brauchen dazu Leitungswasser und etwas Luft, beides haben Sie ja wohl im Hause. An das Wasser schliessen Sie die Zelle an, und mit der Luft ... aber das zeigen wir Ihnen am besten in Funktion, in Ihrem Hause.

Wenn Sie wollen, schicken wir Ihnen unseren Prospekt, aber lieber kommen wir zu Ihnen mit der ganzen Ausrüstung.

Natürlich können Sie auch andere niedermolekulare Substanzen trennen, z.B.: Indole, Porphyrine, Zucker, Purine, Vitamine, anorganische Ionen.

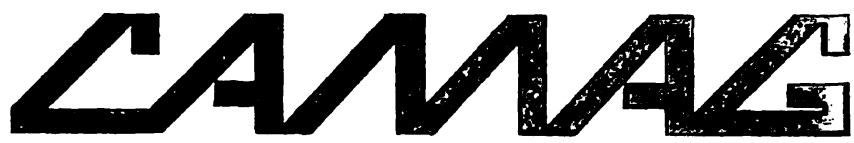

Chemie-Erzeugnisse und Adsorptionstechnik AG Homburgerstrasse 244132 Muttenz/Schweiz

Unser Zweigbetrieb in der Bundesrepublik: CAMAG, 1000 Berlin 45, Baseler Strasse 65 


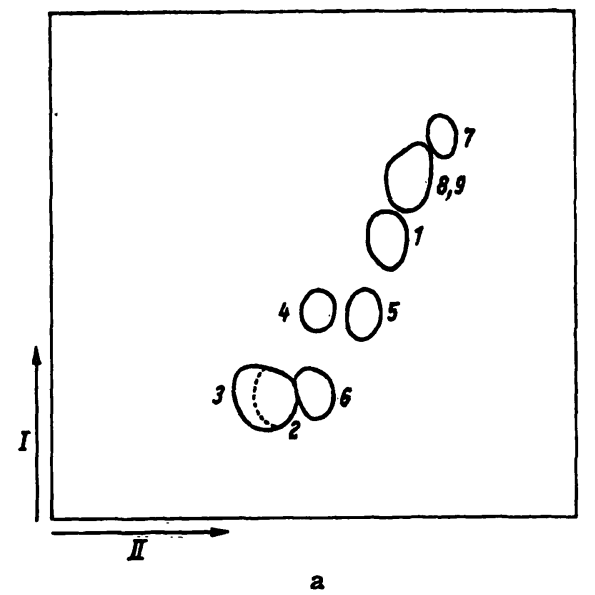

a

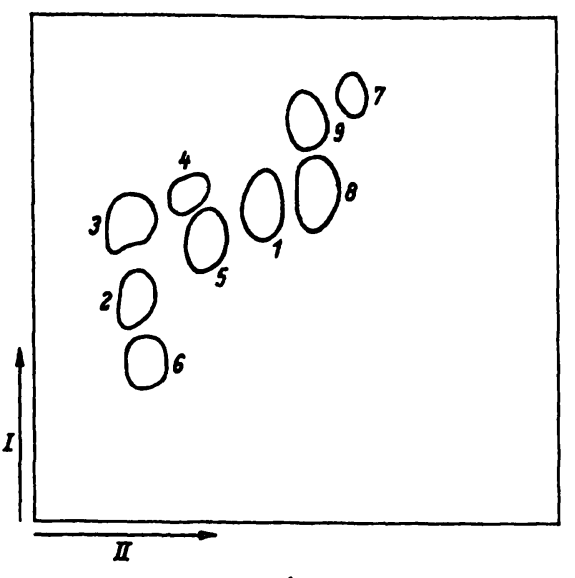

b

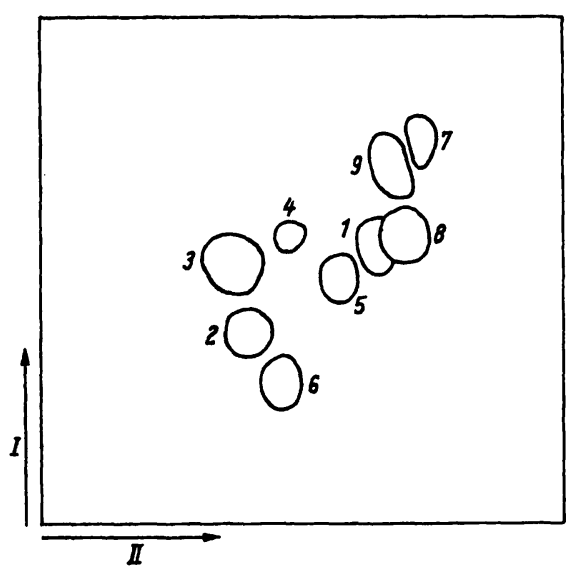

c

Steroidchromatogramme

a) Fließmittelsystem I; b) Fließmittelsystem II ; c) Fließmittelsystem III

1.: Mestranol, 2.: Cyproteron, 3.: Cyproteronacetat, 4.: Progesteron, 5.: Ostron, 6.: Ostradiol, 7.: Ostradiol-dipropionat, 8.: Lynestrenol, 9.: Ethynodioldiacetat

\section{Literatur}

1. Runnebaum B. und J. Zander, Klin. Wscht. 40, 453 (1962). - 2. Graef V. und HJ. Staudinger, diese Z. 5,314 (1967). 3. RöDER, E., Dtsch. Apoth. Ztg. 107, 1007 (1967).

Dr. I. Székács, Abteilungsleiter Staatl. Inst. f. Hygiene, Budapest, IX. Gyáli-ut 2-6, Ungarn 